\section{B A Institute of \\ YK Business Administration \\ 六下 \\ Karachi \\ Leadership and Ideas for Tomorrow}

Business Review

Volume 14 Issue 2 July-December 2019

7-1-2019

\title{
Becoming generous and respecting honor: An experiment based on donation and trust-game with multiple trustees
}

\author{
Gulnaz Anjum \\ Institute of Business Administration, Karachi, Pakistan \\ Werner Gueth \\ Max Planck Institute for Research on Public Goods, Bonn, Germany
}

Follow this and additional works at: https://ir.iba.edu.pk/businessreview

\section{(c) (9)}

This work is licensed under a Creative Commons Attribution 4.0 International License.

\section{Recommended Citation}

Anjum, G., \& Gueth, W. (2019). Becoming generous and respecting honor: An experiment based on donation and trust-game with multiple trustees. Business Review, 14(2), 47-64. Retrieved from https://doi.org/10.54784/1990-6587.1017 


\title{
Becoming generous and respecting honor: An experiment based on donation and trust-game with multiple trustees
}

\author{
Gulnaz Anjum • Werner Gueth
}

\begin{abstract}
This research explores the reoccurring conflict of speculations and explanations among social scientists regarding why people do charity or behave generously. Our participants were 256 subjects, $34 \%$ males and $65.2 \%$ females. Based on a donation task we elicit the most honorable member in a group of four participants. This participant receives a substantial monetary reward and recognition as the most generous donor in the group. An unannounced subsequent phase confronts the honor participant with the task of a single contributor in a best-shot trust game. Without knowing what this honor participant has contributed, the three other participants independently compensate the contributor. We view the contribution level as measuring honor and compensations as measuring respect of honor. With the help of these choice data and additional information from the initial phase as well as from post experimental questionnaires, we assess whether respect of honor reacts monotonically to contributions and how aspects deciding who is honored influence contribution levels and respective compensations. Treatments vary in the (non) linearity of contribution costs. In both treatments we find that honor participants contribute generously to increase their group's welfare, even at the cost of their own profit. Honor participants seem to care for their role and justify their entitlement to this position. Other participants reward respect to honor participants by compensating them.
\end{abstract}

Keywords Honor - Trust game - Multiple trustees - Donation task.

Gulnaz Anjum

Institute of Business Administration, University Road, Karachi-Pakistan

E-mail: ganjum@iba.edu.pk

Werner Gueth

Max Planck Institute for Research on Public Goods, Bonn-Germany

Note: The experimental work of this paper was funded by Max Planck Institute of Economics, Jena Germany. Initial findings of this study were presented at the 28th Annual Convention of Association for Psychological Science, May 21-24, 2015 (New York City, USA). 


\section{Introduction}

It is widely known that people drive happiness when they are involved in charity, mainly due to the feel-good factor that comes with generosity. It is also speculated that we engage in such generous behaviors as it is beneficial for communities at small and societies at large. All social sciences have attempted to find a plausible explanation for why people engage in generous behavior. In this paper we are proposing a reputation and respect based explanation for doing charity or any generous behavior for that matter. For the purpose of operationalization, we propose that this reputation, honor and respect of honor often result from distinguished standards of virtue and courage, possibly when maintained and observed by others over decades as in the case of Nelson Mandela.

In many business and economics frameworks it is assumed that there might be some element of selfishness that drives such generosity (Andreoni 1990). However, recent research has indicated that standard economics theories often fail to support this homo-economicus narrative (Aknin et al 2015, 2012; Dunn et al 2008). It is interesting to note that the evidence that has been documenting the positive associations between populace's general interpersonal trust, also referred to as perceived trustworthiness, reflects on other social indicators of a country such as economic prosperity, health, wellbeing, social equality, and happiness (Espín et al 2016; Kawachi et al 1997; Knack and Keefer 1997; Guiso et al 2008). Despite these macro level analyses (country level), what exactly motivates micro levels (individuals) to act in a trustworthy manner is rather unexplored.

In most experimental and business economics research trust and generosity have been seen as utilitarian. For instance, there is a consensus that trust is not self-less and is a form of self-interest; there is also a sense that it is an expression of a calculated risk based decision that includes one's expectations if others' will be trustworthy or not. Similarly, generosity and trustworthiness have been seen and explored in terms of reciprocity, in such a conception there is an inherent motive of maintaining mutually beneficial relationships (Barkan et al 2015; Espín et al 2016; Hardin 2002; Gambetta 1988).

More in line with experimental psychology are the findings of researchers who suggest that trust and generosity are related to unconditional kindness. They argue for the presence of such a motive that leads to trustworthiness and hence decisions for making contributions for others' benefit. Many studies have confirmed traditional reciprocity as a key reason for trustworthiness (Güth et al 2000; Schotter and Sopher 2006; Bellemare and Kröger 2007; Bornhorst et al 2010). This is apparent in repeatedly found direct relationship between the amount sent by the trustors and the subsequent amounts sent back by the trustees in a given game.

Other researchers also argue that it is because of the intentionality that trustees' trustworthy behavior is in fact also predicted by their own behavior in a standard Dictator Game (Forsythe et al 1994). Meaning that participants who are kind will be trusting even in a Dictator Game, and hence they are likely to be more trustworthy in the trust game as well (Ashraf et al 2006; Chaudhuri and Gangadharan 2007; Kovacs and Willinger 2013). But this does not rule out 
the prevalence of altruistic or egalitarianism related motives in the case when generous offers in the dictator games are triggered by these other motives (Fehr and Schmidt 1999; Iranzo et al 2012).

Much of the literature on honor has the tendency to portray it in a negative light. However as proposed by previous research, honor can have a functional aspect to it when it comes to caring for positive reputational concerns. For instance, in a set of studies conducted in Germany, Pakistan, South Korea and USA, Anjum (2016) has shown that people express more respect for members who follow norms of respectability. Such increased expression of respect was based on whether people were able to contribute socially, the more they contributed, the more they became relevant for gaining prestige. Hence it was concluded that social contribution and adherence to honor norms lead to honor. Furthermore, it was shown that people did not have to be ingroup members for monitoring adherence to honor codes. These codes are recognized and observed if someone wants to be on the pedestal of being honorable (Anjum et al 2019).

Finally, there is a lack of research on the conscious emotions that is to say, how these emotions effect people's choices and decisions. This might be interesting to find out what, on a conscious level, drives these episodes of charity and generosity. We propose that out of the two conscious emotions of shame and guilt, shame which is mostly associated with gaining honor or losing honor and respect. For this study we define honor as respect and distinction gained via relatively high standards acquired in a given group. In the lab environment we are going to study:

- selecting an honorable participant out of a small group and informing the other group members what qualifies their "honor participant"

- providing a chance for the "honor participant" to actually prove his or her social responsibility

- allowing the other group members to show their respect by monetarily compensating their "honor participant"

- a single contributor and the best-shot technology (all gain equally from just one contribution, namely the contribution of the "honor participant")

- an additional reward phase allowing the other group members to reward their "honor participant".

One can compare this with trust or, more generally, with reciprocity experiments. The special features of this study would be that there are more than one, actually three, trustees and partly non-linear contribution costs. The former allows for an excuse of freeriding in compensating by arguing that others will sufficiently respect "honor". Due to the latter, the non-linear costs, we can distinguish noise from trading off own and social concerns since the 'honor participant' can contribute less than individually and more than socially desirable.

Hence, we will explore how contributing and respect of honor reacts to such phenomena. The process of selecting the most honorable member, experimentally implemented, is line with usual practice in the field (Nobel laureates are widely honored but also receive large prizes, the same applies to Olympic champions). Such monetary prize is an additional aspect of our honor ceremony in the lab. Naturally, both prize money and social recognition are minor compared to rewards in the field. But in the lab it also does not require a lifetime in the 
lab to become honorable and to respect honor i.e. the lab downscales both the difficulty of proving one's honor and what one gains from honor. The advantage of the stylized lab structure is, of course, control of aspects which can hardly ever be assessed in the field like: i) the cost involved when proving one's honor; ii) the information about the honor participant; an obvious intuition about one's solidarity with group.

This study proposes a unique way of role allocation, especially of selecting the 'honor participant', is expected to generate higher face validity. People feel good by acts of giving (Andreoni 1989, 1990). We allow them to display their generosity in a donation task. To motivate donations in the lab we double the donations and also offer them receipts of actual transactions. Previous research also recommends that use of multiple players for trust game could be more beneficial for eliciting social motives in the lab environment (Espín et al 2016). Espín et al (2016), argue that having two players in the game brings variation in their social motives. In their framework, subjects elicit trustworthiness that can be used to infer complex motivations. Therefore, we have developed a specific paradigm for a trust game with multiple trustees.

Herein participants, who have earned their role, surprisingly receive an additional amount of money. This would give them the sense of deservingness and that they are entitled to own this money. There are robust findings indicating that people are less likely to share an earned income, compared to sharing 'manna from heaven' (Ruffle 1998; Cherry and Shogren 2008; Carlsson et al 2009). Therefore, if participants still share their fortune this supports our interpretation that "honor" participants care for their role and position.

Previous research has shown that non-monetary acknowledgement can stimulate contributions (although there is faster decline compared to monetary sanctions; Masclet et al (2003)). Hopefully the combination of non-monetary appreciation (social recognition and responsibility for the group) and monetary appreciation, the additional "honor" prize, allow to experimentally assessing what determines proving one's honor and respecting honor.

\subsection{The game model}

How the most honorable participant $h$ in a group of four participants is selected will be described in the following section. Assume for the beginning that $h$ is already selected. One aspect of honoring $h$ is that $h$ receives a considerably larger monetary endowment $E$ (actually $E$ is composed of the same positive show up fee $e$ for all participants whereas $E-e$ is given to $h$ as the "honor prize"). After having endogenously selected $h$ the game process continues with two stages:

- first $h$ chooses a voluntary contribution $c$ satisfying $0 \leq c \leq E$ about which all other group members $i \neq h$ are informed before

- all three other group members $i \neq h$ determine their monetary transfer $t_{i}$ to $h$ where $t_{-} \leq t_{i} \leq e$.

The monetary payoffs in case of linear contribution costs, the $L$ treatments, are 
$-E-c+\alpha_{L} c+\sum t_{i}$ for $h$ and

$-e+\alpha_{L} c-t_{i}$ for all $i \neq h$

The parameter $\alpha$ is such that $0<\alpha_{L}<1<4 \alpha_{L}$ as usual in linear public goods games (see Ledyard, 1995, for an early sway): $h$ suffers an own monetary loss when increasing $c$ but the group of all four participants collectively gains. Actually, in the $L$ treatments, we set $\alpha_{L}=0.6$. Rather than a best-shot public goods game with a preselected only contributor, one can view the interaction as a trust game with

- the honor participant $h$ as the trustor and

- three trustees $i \neq h$.

Compared to usual gift exchange games, for example, representing principalagent relationships (Fehr et al 1993) it depends on $t_{-}$whether one can only reciprocate by gifts i.e. in case of $t_{-}>0$ or, whether in case of $t_{-}<0$, also by punishing, e.g. in case of $c=0$. Actually, we assume $E=4 e$ so that when $h$ chooses $c=E$ and each $i \neq h$ compensates $h$ by $t_{i}=e$, the resulting payoffs are

$-\alpha E+3 e=e(2.4+3)=5.4 e$ for $h$ and

$-\alpha E=4 \alpha e=2.4 e$ for each $i \neq h$

Thus, $h$ maintains his endowment advantage of $E-e=3 e$ over the three others and each of the four group members gain 1.4e by efficient voluntary public good provision and compensations by $i$ participants. The only variation of treatment $L$ concerns $t_{-}$which is

- once $t_{-}=0$, the gift exchange treatment $L_{0}$, and

- once $t_{-}=e$, the potential respect treatment allowing the three $i \neq h$ participants to give money to $h$.

What differs in the $N$ treatments are the non-linear contribution costs and the adapted parameters $e_{N}(>0)$ and $\alpha_{N}(>0)$. Contributing in treatments $N$ costs $c 2 / 2$ rather than $c$. Thus what $h$ earns is given by $E_{N}-c^{2} \div+\alpha_{N} c+\sum t_{i}$. In case of general monetary opportunism so that $t_{i}^{*}=t_{-}$for $i \neq h$ an opportunistic $h$ participant, anticipating these $t_{i}^{*}$ choices, would choose $c^{*}=\alpha_{N}$ whereas maximizing the total earnings of all four group members, namely $3 e_{N}+E_{N}-$ $c^{2} \div 2+4 \alpha_{N} c$, requires $c^{+}=4 \alpha_{N}$.

Since we assume $E_{N}>4 \alpha N$, treatments $N$ allow to contribute less than optimal and more than efficient what is excluded by $L$ treatments for which $c^{*}=0$ and $c^{+}=E$. If honor inspires unreasonable contributions $c \epsilon\left[c^{*}, c^{+}\right]$we can explore how respect of honor, measured by $t_{i}$ choices of $i \neq h$, reacts to such contributions. In our view, the $N L$ treatment should induce interior choices in the range $c_{i}^{*}<c_{i}<c_{i}^{+}$whereas the $L$ treatments trigger more "bang-bang" behavior, $c \epsilon\left\{c^{*}, c^{+}\right.$. Via treatment-specific parameters one can guarantee the same efficiency gains of $c^{+}$rather than $c^{*}$ for both treatments what requires $\alpha_{N}^{2}=2\left(e_{L}-e_{N}\right)$. Imposing we additionally ensured equal total earnings for both treatments, $L$ and $N$, in case of $s *$.

Business Review: (2019) 14(2):47-64 


\section{Method}

2.1 Exploratory research questions and hypotheses

Will 'honor' enhance efficiency and rewarding? We will try to answer this experimentally as well as the more specific research questions:

- Do honor contributors, $h$ participants, contribute more or less than rational or more or less than socially optimal?

We speculate that $h$ participants contribute more to maintain and enhance their prestige and their group's welfare.

- Are the other group members, $i$ participants, more opportunistic or reciprocating?

Those, who perceive the selection of $h$ as procedurally fair, based on contributing to a charity, may make higher compensations to $h$ participants (indicating respect and appreciation). Those, who perceive the selection of $h$ as procedurally unfair, based on contributing to a charity or may not value charity, may compensate less to $h$ participants indicating jealousy and envy. The benchmark solution, predicting $t i *=t_{-}$for all $i \neq h$ and $c=c *$ in both treatments, assumes common and, on behalf of $h$, also anticipated monetary opportunism, i.e. each group member is only concerned about their own monetary gain. This is not what we expected and also not what we observed. What we predicted and confirmed is that:

- honor participants $h$ care for their honor and

- I participants appreciate and reward honor, i.e. respect honor by paying dearly.

Such concerns are by their very nature idiosyncratic and private i.e. $h$ cannot know how much other participants respect honor, especially since we only implemented a one shot interaction, which excluded to learn what motivates $i$ participants. For our experimental set-up assuming common knowledge of what motivates $h$ and what drives respect of honor by $i$ participants would at best yield an ex-post rationalization and only transform the question 'why such behavior?' into asking 'why such commonly known concerns?'. Only such ex-post justification would apply to many paradigms, e.g. most social dilemma games, this should hint upon information about the psychological mechanisms generating such behavior.

\subsection{Sample}

The participants were recruited using an online invitation portal, Online Recruitment System for Economic Experiments (ORSEE by Greiner (2004)) available at Max Planck Institute of Economics, and Friedrich-Schiller University of Jena, Germany. We ran 8 sessions with 32 participants each. Each participant qualifies as an independent observation. Of the 256 subjects, 128 participated in each treatment: linear and non-linear. For the choice data collected in linear and non-linear session's average earnings amounted to $9,18(\mathrm{SD}=1.22)$ and $19,34(\mathrm{SD}=1.53)$, including a show up fee of 2,50 (also see figure 2,3 ) 
what already indicates strong demonstration for benchmark behavior. Regarding gender composition there were $34 \%$ males and $65.2 \%$ females. There were more women than men in each session.

\subsection{Experimental protocol and procedure}

The experiment consists of a pre-phase, followed by the main phase. In the prephase all participants are endowed with 5 (in addition to their show-up fee) and asked how much of it they would donate to a self-selected charity. Whatever amount out of their 5 endowment they choose to donate would be doubled ${ }^{1}$ and transferred to their chosen charity. Based on their intended donations among 32 participants the 8 highest donors are selected as honor participants $h$ whereas the remaining 24 participants are identified as other participants $i$. Only what the $h$ participants donate is actually doubled and transferred to the charity of their choice i.e. the donations by the other $i$ participants are purely hypothetical and not payoff relevant. The donations by $h$ participants are deducted from their 5,00 endowments.

In each group of four participants one honor participant $h$ and three other participants $i$ learn their roles, based on the donations in the pre-phase. $h$ participants are additionally endowed with 20 ECU and asked for their contribution $c$. In the next step $i$ participants, unaware of their earnings from $h$ 's contributions, get $5 \mathrm{ECU}$ and decide how much of it they would like to transfer to their $h$ participant. After that the payoffs of the four participants from the trust game and their earnings are calculated and made public.

At the end of experiment all participants answer a questionnaire about their motivation and some demographics. Participants are paid what they kept in the pre-phase and what they earned in the main experiment in addition to their show-up fee. They are also asked if they like to have a debriefing on the experiment via email and whether they want to be informed about actual money transfer to the charities (twice the donations made by $h$ participants). The experiment was conducted with the help of Z-tree (Fischbacher 2007).

\section{Results}

IBM SPSS 23 was used for all screening and analyses. Initial screening of all data points was done before conducting all analyses using various descriptive and comparative analyses such as ANOVA and regressions. In the first task (the donation task), which is the same for all participants, two choices were most popular: to give nothing to any charity (47.30\% of all subjects) and to give everything to the self-selected charity (12.50\%). There are no gender differences in average donation: mean donation by males and females are 1,36 (SD = 1.85 ) and $1,35(\mathrm{SD}=1.66)$, respectively. Figure 5 (see Appendix) summarizes

\footnotetext{
1 This avoids the possible excuse of donating afterwards, i.e. feel warm glow for themselves by giving outside and avoiding involvement of experimenters.
} 


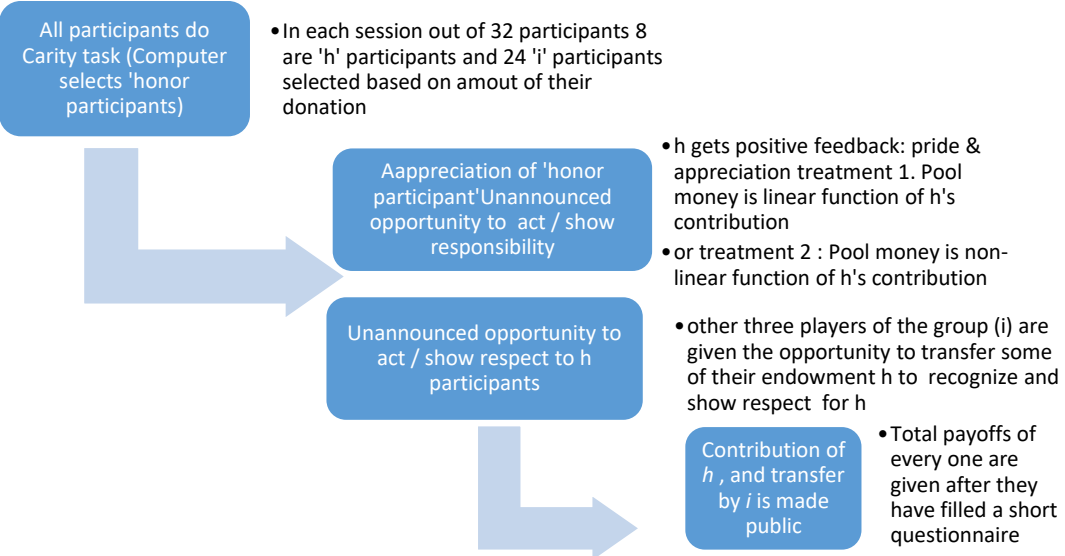

Fig. 1: Flow of experimental protocols

percentages for all choices. It is also interesting that, in spite of heightened concerns for Ebola, the NGO Doctors Without Borders was rarely chosen. Figure 4 (see Appendix) summarizes percentages of donation choices.

Regarding the main phase two important results answer our specific research questions. The first result concerns the question whether participants in $h$ roles contribute more (or less) to maintain and enhance their prestige and their group's welfare. As expected contributions in linear $(\mathrm{M}=7.88, \mathrm{SD}=6.70)$ and non-linear $(\mathrm{M}=19.63, \mathrm{SD}=1.07)$ treatments are significantly different $(\mathrm{t}$ $=9.79, \mathrm{p}<0.001): h$ participants in linear treatment display a wider spread distribution (see appendix figure 6 ). Less than a quarter of all $h$ subjects $(21.88$ $\%$ ) contribute zero and $50 \%$ contribute between half and everything; the modal choice is contributing $10 \mathrm{ECU}$ ( $25 \%$ of subjects). These findings support that $h$ participants feel responsibility for their group's welfare. Giving everything to pool money, meaning to give 40 percent of what they have got, accounts for $12.5 \%$ participants.

In the non-linear treatment, except for 4 subjects, who contribute 60 or 80 percent, everyone contributed everything, which can be justified by efficiency concerns. This rules out that after being honored, $h$ subjects try to punish low donors. These findings altogether suggest that honored people act responsibly and care a lot for their group's welfare.

Our second set of findings are based on data of the second stage where $i$ subjects can express respect for $h$ participants via compensating. In both conditions a significant proportion of $i$ subjects reward their $h$ participants indicating respect of honor. In (non)linear treatment $32.80 \%(43.30 \%)$ of $i$ subjects choose compensations between 0.5 and $3 \mathrm{ECU}$ (see figure 3 ) $^{2}$. On average in the linear and non-linear treatment $i$ participants give $0.48 \mathrm{ECU}(\mathrm{SD}=0.79)$ and 0.86 ECU $(\mathrm{SD}=1.27)$, respectively. The significant difference in mean compensations in two conditions $(t=2.88, p<0.01)$ might be due to the higher payoffs in the non-linear treatment. In both conditions participants clearly respect honor

\footnotetext{
2 Note that $i$ participants compensate after $h$ having contributed i.e. they do not compensate in order to trigger a higher contribution by $h$
} 
via compensating.

To explore causal relationship between contribution by $h$ and compensations by $i$ participants we ran a regression analysis to learn if compensations depend on contributions. In both treatments compensations by $i$ participants depend significantly on $h^{\prime} s$ contribution. For the non-linear treatment dependency (linear regression: standardized $\beta=-0.39, t=4.78, p<0.001$ ) is stronger than for the linear treatment (standardized $\beta=-0.25, t=2.91, p<0.01$ ). Thus compensations by $i$ participants react positively to how responsibly $h$ participants behave.

Other findings are based on the post experimental questionnaire, to assess a participant's personal guilt and shame evaluations and related repair or withdrawal tendencies associated with these two attributes. Donations by subjects show a positive but a weak association with repair tendency of participants $(r=0.12, p<0.05)$ : participants tend to avoid guilt feelings by donating. For $h$ participants of the linear treatment there are no significant relations between any of these attributes and contributions (see the correlation matrices in table $1)$.

Furthermore, in the non-linear treatment there is a strong association between contributions of $h$ participants and their perception of shame based withdrawal. This indicates that when $h$ participants earn more as in the non-linear treatment, they would have felt shame when not contributing: contributions significantly depend on withdrawal tendency (linear regression: standardized $\beta=-0.334, t=1.94, p=0.06$ ). None of the four attributes (guilt and shame evaluations and repair or withdrawal tendencies) has any significant influence on compensations by $i$ participants. In our view, this may be due to the fact that three $i$ participants share the responsibility to respect honor that can be expected to weaken the influence of individual guilt and shame.

\section{Discussion}

The experimental decision process begins by endogenously selecting the most honorable member for each group with four participants each. This honor person receives a substantial monetary prize as well as an appraisal for being most generous. Then each honor participant acts as the unique trustor facing multiple (three) trustees. Each honor participant is the only contributor, is awarded the role of being the trustee for their group and plays a trust game with multiple dependents. The honor participant thus acts in this task as a single contributor of the best-shot public good game and can be monetarily rewarded by the other three group members. This set-up allows for voluntary cooperation but also free riding in respecting honor.

Our findings suggest that honor participants care for their group's welfare by contributing to the public good. They like to share their honor prize and want to maintain their reputation obtained in the initial donation task. This clear sense of entitlement to honor and attempt to maintain this entitlement has direct implications for group functioning and organizational leadership. When saliently recognized and respected, people care more for their group even when 
this is costly for themselves. Honor apparently plays an important role in motivating individuals to do well e.g. by enhancing their group's welfare.

Furthermore, not only the honor participants act more responsibly when saliently recognized, but also other participants in their groups show respect by compensating: most of the three other participants independently compensate the contributor, even though they receive only one fourth the honor prize. We conclude that this compensation from $i$ participants to their honor participant shows respect of honor. In our view, these findings have important implications for group performance and perceived fairness in organizational settings: followers, e.g. "trustees" or "subordinates" will respect those who have legitimately earned their position or title.

Concerning implications for fundraising it seems that, first of all it is true that people feel better by the act of giving (Andreoni 1989, 1990). What holds back student participants, who usually consider themselves as needy, donating in the lab, was managed by doubling their donations for self-selected charities. Using quite popular charities gave another motivational buzz to donate. In our view using mentally available charity organizations and incentivizing (by doubling) will induce more donations from participants. There is evidence (Cherry and Shogren 2008; Carlsson et al 2009) suggesting that, when participants have earned their endowment, they are less likely to give it away. But saliently appraising, like in our study, motivates people to give.

The respect of the honorable participants for their generosity is in line with the research that indicates that honor is a matter of social reputation (Cohen and Nisbett 1994; Cohen et al 1996; Cohen and Nisbett 1997; Cross et al 2013). They mainly argue that people retain honor by warding off insults and protecting their social image in front of others. Such concerns to follow honor values are seen across cultures (Guerra et al 2013). This has important implications for the self-esteem of the person as they want to categorize themselves as respected members of their group (Ellemers et al 1999). This experiment has not taken the route related to inducing insults in the lab but it has raised reputational concerns in the group and lab setting. It can be inferred that even when people's identity is hidden, they still want to be honorable and be respected.

This points to a lack of research on the conscious emotions and how these conscious emotions affect people's choices and economic decisions. Therefore, this study has meaningfully explored this on a conscious level drive of charity and generosity. In this respect, the two conscious emotions of shame and guilt were explored and it is possible to state that shame is rightly mostly associated with gaining honor or losing respect (Anjum 2016). Hence the unique findings of this study regarding game theory based contributions and emotional associations (shame and guilt) have additional significance.

In the previous research, generosity has been seen in relationship with trustworthiness and explored in terms of reciprocity, whereby there is the motive of maintaining mutually beneficial relationships (Barkan et al 2015; Espín et al 2016; Hardin 2002). But such an exploration with conscious emotions is missing from research. For instance, previous research has indicated that there are multifaceted possibilities of motivations for generosity (in this case being honorable through being generous). This research adds to this literature that the 
association of the possibility of felt shame may be the stronger motive behind generosity and feeling honorable than the motive of guilt.

Although previous studies have confirmed that reciprocity is the main reason for trustworthiness (Güth et al 2000; Schotter and Sopher 2006; Bellemare and Kröger 2007; Bornhorst et al 2010) but the impact complex emotions such as guilt or shame have on trustworthiness has not been explored in the previous research. Generous reciprocity is seen in most games, it is even apparent in the amount sent by the trustors and the subsequent amounts sent back by the trustees in a given game. But the emotional significance of guilt and shame is missing from this literature. Hence, this research bridges this gap in line with experimental psychology and experimental economics by offering an additional motive based on conscious emotions by suggesting that generosity is not only related to unconditional kindness it may be related to respect related reciprocity as well.

The findings of this research argue for the presence of a motive related to shame, a motive that may lead to drive for active generosity in order to gain respect and hence decisions for making contributions for others' benefit the person. In line with psychological research on honor (Anjum 2016) this research points to the intentionality that trustees' trust worthy behavior. This also attempts at answering some of the concerns raised by previous literature by showing that there is a possibility of ruling out the prevalence of altruistic or egalitarianism related motives (Fehr and Schmidt 1999; Iranzo et al 2012).

\subsection{Implications and limitations}

This research has key implications for policies and advocacies for fundraising and corporate social responsibility. Most of the western literature has set out a negative stance on honor. This paper has conceptualized honor as an assignment of recognition for being a member of a prestigious group. Members of honor groups are obliged to follow their group's honor code; not sticking to the honor code threatens reputation and instigates condemnation. As indicated by Anjum (2016) honor has a functional role as norms related to honor codes are recognized and observed if someone wants to be on the pedestal of being honorable. In this lab experiment, it is established that when someone is declared honorable, they have a higher tendency to contribute more generously in the next rounds of the game. These findings replicate and confirm the assumptions of honour proposed by Anjum et al (2019).

In future research, one might consider situations with more than one honor participant, who for example, could jointly enhance their group's welfare as the only contributor either based on the weakest-link technology ${ }^{3}$ or that one of linear public goods games. Would presence of other honor participants crowd out feeling responsible for one's group e.g. in the sense of bystander effect due to diffused responsibility (Darley and Latané 1968)? Would $i$ participants respect honor more or less when there are more honor participants, contributors to

\footnotetext{
3 The minimum of all contributions determined the level of public good from which all group members, contributors as well as all other group participants profit equally.
}

Business Review: (2019) 14(2):47-64 


\section{G. Anjum, W. Gueth}

the public good based for instance on the weakest link technology (Leary and Forsyth 1987)?

One key limitation of this study lies in the assumption that it has focused only on the motives of honor which is more of a behavioral component and then it is being compared with cognitive aspects such as shame and guilt. As previous research has shown that motives are complex, especially heterogeneous motives are more likely in trust games such as the one employed in this experiment. Also Espín et al (2016) have argued that even in the two players game there is dramatic variation in the motives and these motives are particularly social when it comes to the trust game. This also adds heterogeneity that is non-negligible. In this experiment the implications of these findings are further squeezed because of two elements that we did not control for i.e. self-interest of the participants and their drive for efficiency considerations.

Also our research does not show if participants expressed antisocial motives such as spitefulness. Former research has shown that individuals are not solely kind they are also capable of expressing spite in addition to selfishness and would send zero offers to others (Brañas-Garza et al 2014). This research hence does not capture that lack of kindness and the motives engaged on a mental or behavioral level.

\section{Conclusion}

The topic explored in this paper answered two significant questions. First, does becoming generous induce honor among other ingroup members? Second, do people respect the generosity of others in their group and whether people respond with respect towards the generous group members? These two questions have been heavily debated in experimental economics and business psychology but have not been tested in cross-disciplinary experiments. Therefore, this study provides an insight into this reoccurring conflict of speculations among social scientists regarding why people do charity or behave generously. In fact, to become honorable and earn respect from others is the answer.

Based on an induced donation task in the lab, we elicited the most honorable members in their respective groups of four participants. We viewed the contribution level as honor and compensations as respect of honor. We conclude that honor participants contribute generously to increase their group's welfare, even at the cost of their own profit. Furthermore, honor participants seem to care for their role and justify their entitlement to their position of becoming honorable. Other participants reward respect to honor participants by compensating them. These findings have implications for charity, leadership, and various social psychological aspects associated with respect and honor.

\section{References}

Aknin LB, Dunn EW, Norton MI (2012) Happiness runs in a circular motion: Evidence for a positive feedback loop between prosocial spending and happiness. Journal of Happiness Studies 13(2):347-355 
Aknin LB, Broesch T, Hamlin JK, Van de Vondervoort JW (2015) Prosocial behavior leads to happiness in a small-scale rural society. Journal of Experimental Psychology: General $144(4): 788$

Andreoni J (1989) Giving with impure altruism: Applications to charity and ricardian equivalence. Journal of political Economy 97(6):1447-1458

Andreoni J (1990) Impure altruism and donations to public goods: A theory of warm-glow giving. The economic journal 100(401):464-477

Anjum G (2016) Cross-national assignment of honor: Assignment of honor in germany, pakistan, south korea, and the usa. PhD thesis, Thüringer Universitäts-und Landesbibliothek Jena

Anjum G, Kessler T, Aziz M (2019) Cross-cultural exploration of honor: Perception of honor in germany, pakistan, and south korea. Psychological Studies pp 1-14

Ashraf N, Bohnet I, Piankov N (2006) Decomposing trust and trustworthiness. Experimental economics 9(3):193-208

Barkan R, Ayal S, Ariely D (2015) Ethical dissonance, justifications, and moral behavior. Current Opinion in Psychology 6(DEC):157-161

Bellemare C, Kröger S (2007) On representative social capital. European Economic Review 51(1):183-202

Bornhorst F, Ichino A, Kirchkamp O, Schlag KH, Winter E (2010) Similarities and differences when building trust: the role of cultures. Experimental Economics 13(3):260-283

Brañas-Garza P, Espín AM, Exadaktylos F, Herrmann B (2014) Fair and unfair punishers coexist in the ultimatum game. Scientific reports 4:6025

Carlsson F, He H, Martinsson P (2009) Easy come, easy go-the role of windfall money in lab and field experiments. rapport nr: Working Papers in Economics 374

Chaudhuri A, Gangadharan L (2007) An experimental analysis of trust and trustworthiness. Southern Economic Journal pp 959-985

Cherry TL, Shogren JF (2008) Self-interest, sympathy and the origin of endowments. Economics Letters 101(1):69-72

Cohen D, Nisbett RE (1994) Self-protection and the culture of honor: Explaining southern violence. Personality and Social Psychology Bulletin 20(5):551-567

Cohen D, Nisbett RE (1997) Field experiments examining the culture of honor: The role of institutions in perpetuating norms about violence. Personality and Social Psychology Bulletin 23(11):1188-1199

Cohen D, Nisbett RE, Bowdle BF, Schwarz N (1996) Insult, aggression, and the southern culture of honor: An" experimental ethnography.". Journal of personality and social psychology 70(5):945

Cross SE, Uskul AK, Gerçek-Swing B, Alözkan C, Ataca B (2013) Confrontation versus withdrawal: Cultural differences in responses to threats to honor. Group Processes \& Intergroup Relations 16(3):345-362

Darley JM, Latané B (1968) Bystander intervention in emergencies: Diffusion of responsibility. Journal of personality and social psychology $8(4 \mathrm{p} 1): 377$

Dunn EW, Aknin LB, Norton MI (2008) Spending money on others promotes happiness. Science 319(5870):1687-1688

Ellemers N, Kortekaas P, Ouwerkerk JW (1999) Self-categorisation, commitment to the group and group self-esteem as related but distinct aspects of social identity. European journal of social psychology 29(2-3):371-389

Espín AM, Exadaktylos F, Neyse L (2016) Heterogeneous motives in the trust game: a tale of two roles. Frontiers in psychology 7:728

Fehr E, Schmidt KM (1999) A theory of fairness, competition, and cooperation. The quarterly journal of economics 114(3):817-868

Fehr E, Kirchsteiger G, Riedl A (1993) Does fairness prevent market clearing? an experimental investigation. The quarterly journal of economics 108(2):437-459

Fischbacher U (2007) z-tree: Zurich toolbox for ready-made economic experiments. Experimental economics 10(2):171-178

Forsythe R, Horowitz JL, Savin NE, Sefton M (1994) Fairness in simple bargaining experiments. Games and Economic behavior 6(3):347-369

Gambetta D (1988) Trust: Making and breaking cooperative relations

Greiner B (2004) An online recruitment system for economic experiments

Business Review: (2019) 14(2):47-64 
Guerra VM, Giner-Sorolla R, Vasiljevic M (2013) The importance of honor concerns across eight countries. Group Processes \& Intergroup Relations 16(3):298-318

Guiso L, Sapienza P, Zingales L (2008) Trusting the stock market. the Journal of Finance 63(6):2557-2600

Güth W, Königstein M, Marchand N, Nehring K (2000) Trust and reciprocity in the investment game with indirect reward. Tech. rep., Discussion Papers, Interdisciplinary Research Project 373: Quantification

Hardin R (2002) Trust and trustworthiness. Russell Sage Foundation

Iranzo J, Floria LM, Moreno Y, Sanchez A (2012) Empathy emerges spontaneously in the ultimatum game: small groups and networks. PloS one 7(9):e43,781

Kawachi I, Kennedy BP, Lochner K, Prothrow-Stith D (1997) Social capital, income inequality, and mortality. American journal of public health 87(9):1491-1498

Knack S, Keefer P (1997) Does social capital have an economic payoff? a cross-country investigation. The Quarterly journal of economics 112(4):1251-1288

Kovacs T, Willinger M (2013) Are trust and reciprocity related within individuals? The BE Journal of Theoretical Economics 13(1):249-270

Leary MR, Forsyth DR (1987) Attributions of responsibility for collective endeavors.

Masclet D, Noussair C, Tucker S, Villeval MC (2003) Monetary and nonmonetary punishment in the voluntary contributions mechanism. American Economic Review 93(1):366-380

Ruffle BJ (1998) More is better, but fair is fair: Tipping in dictator and ultimatum games. Games and Economic Behavior 23(2):247-265

Schotter A, Sopher B (2006) Trust and trustworthiness in games: An experimental study of intergenerational advice. Experimental Economics 9(2):123-145

\section{Appendix}

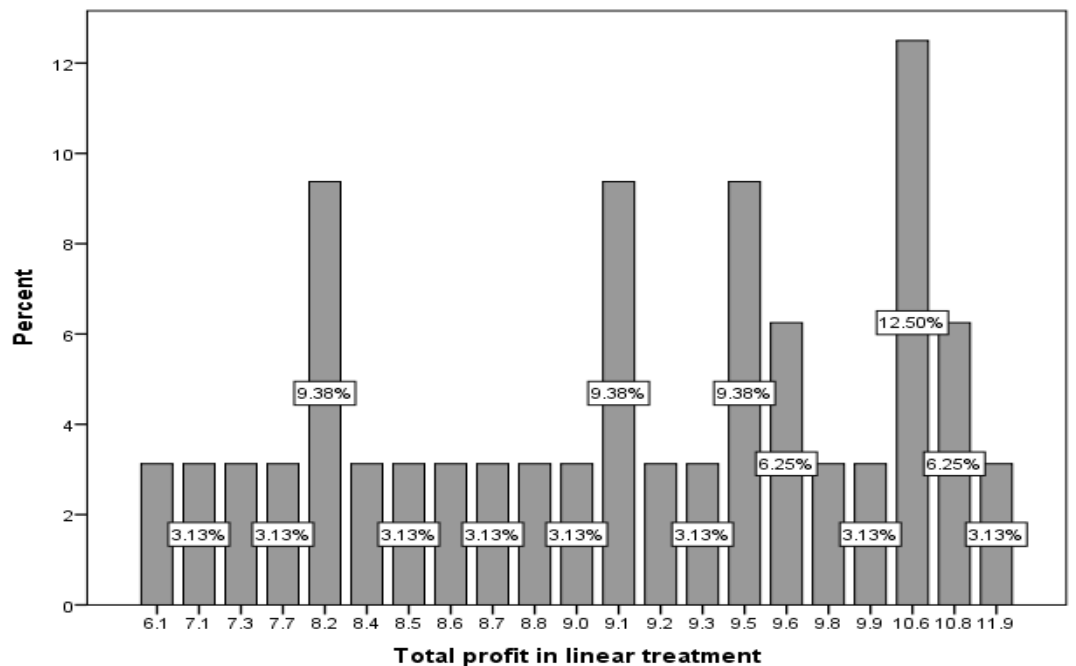

Fig. 2: Total profit in linear treatment 


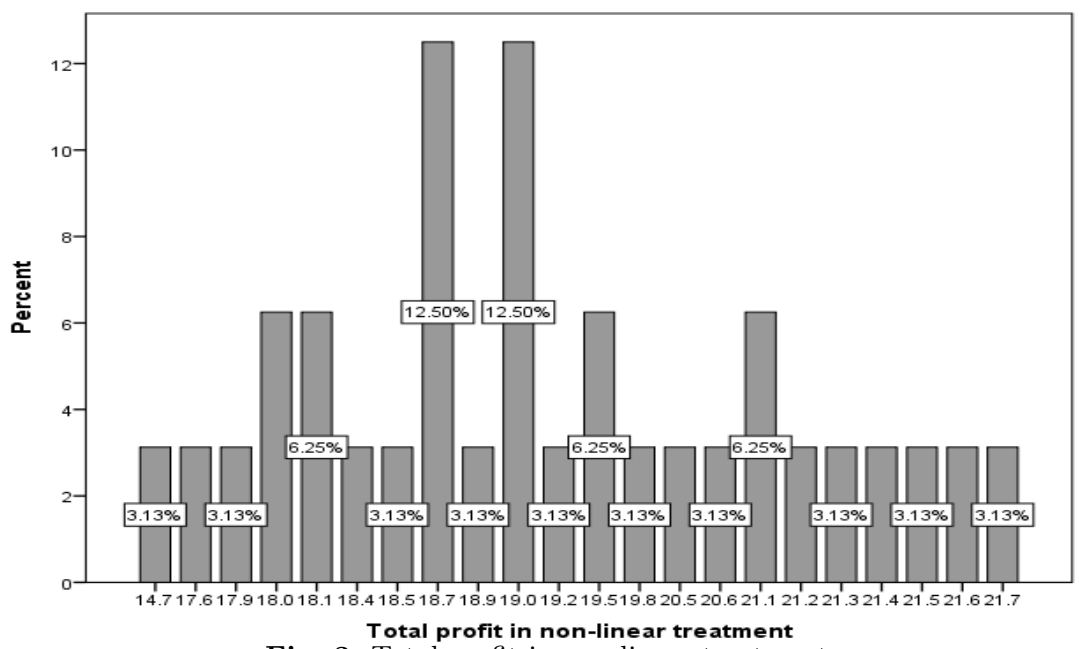

Fig. 3: Total profit in non-linear treatment

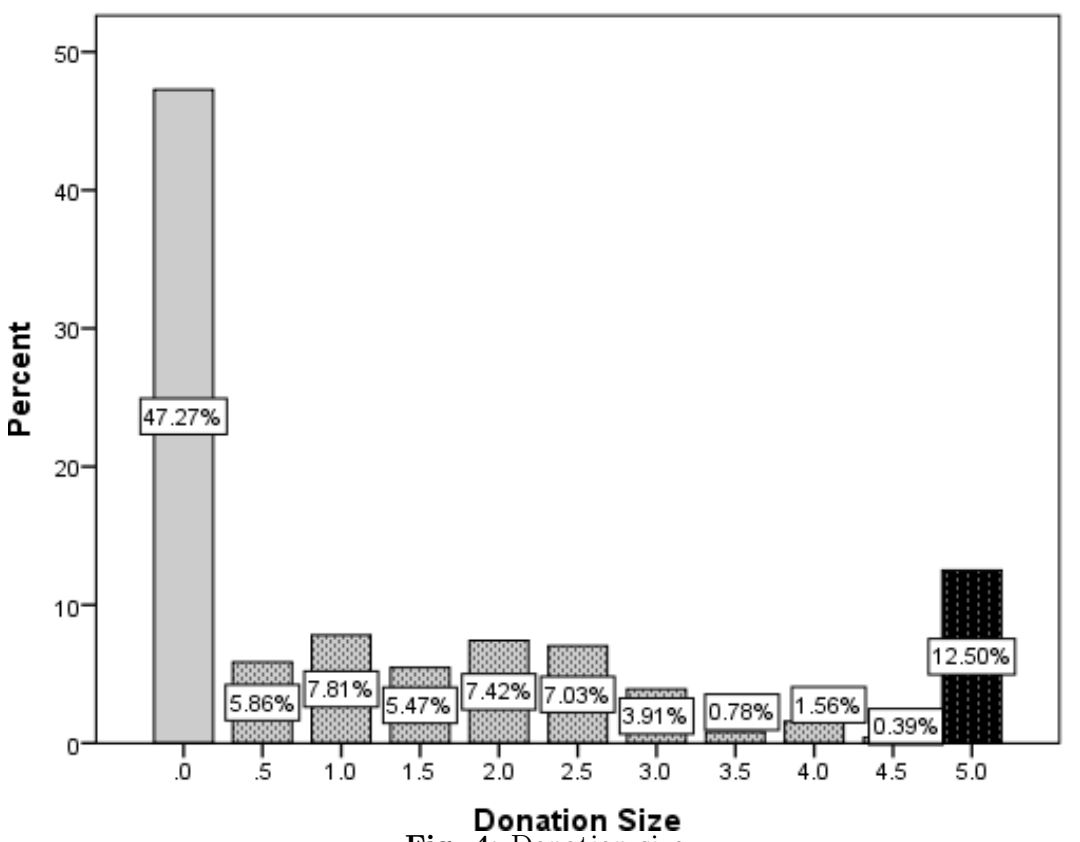

Fig. 4: Donation size 


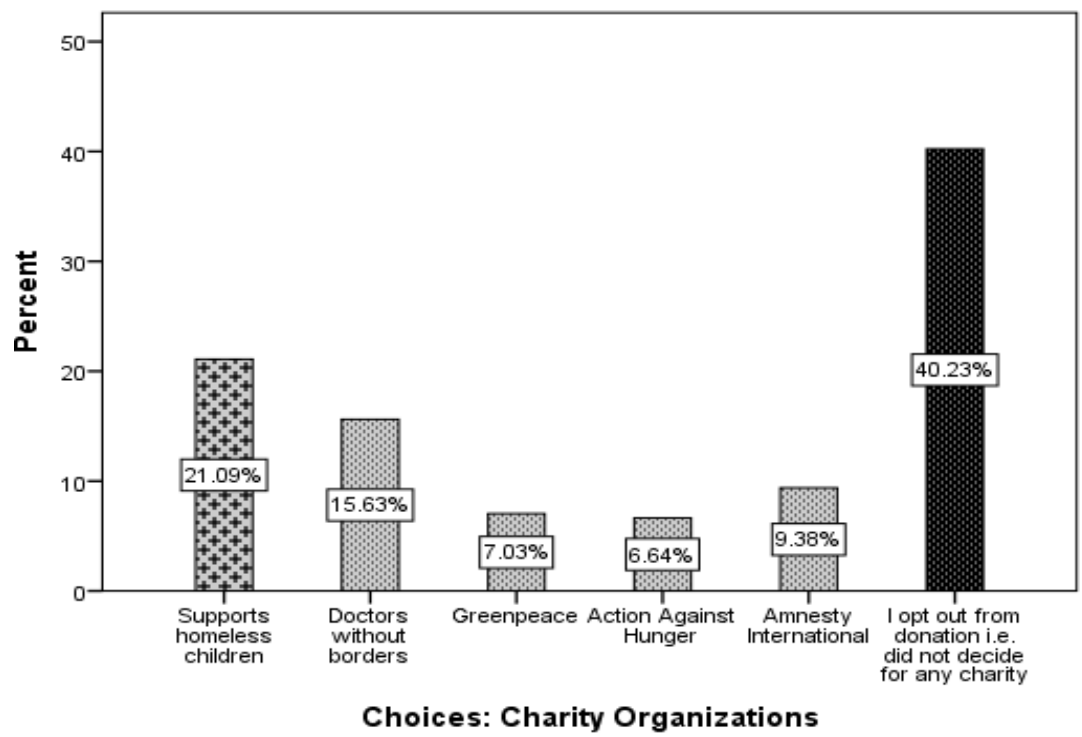

Fig. 5: Choices: Charity organizations

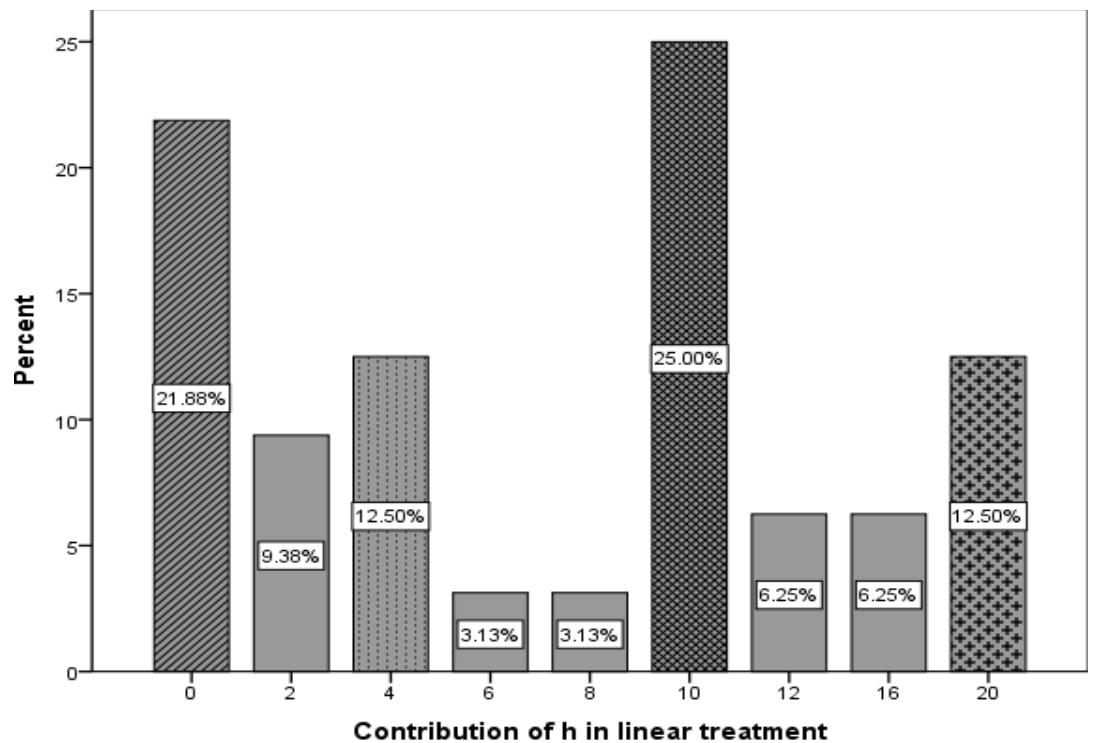

Fig. 6: Contribution of $\mathrm{h}$ in linear treatment 
Becoming generous and respecting honor...

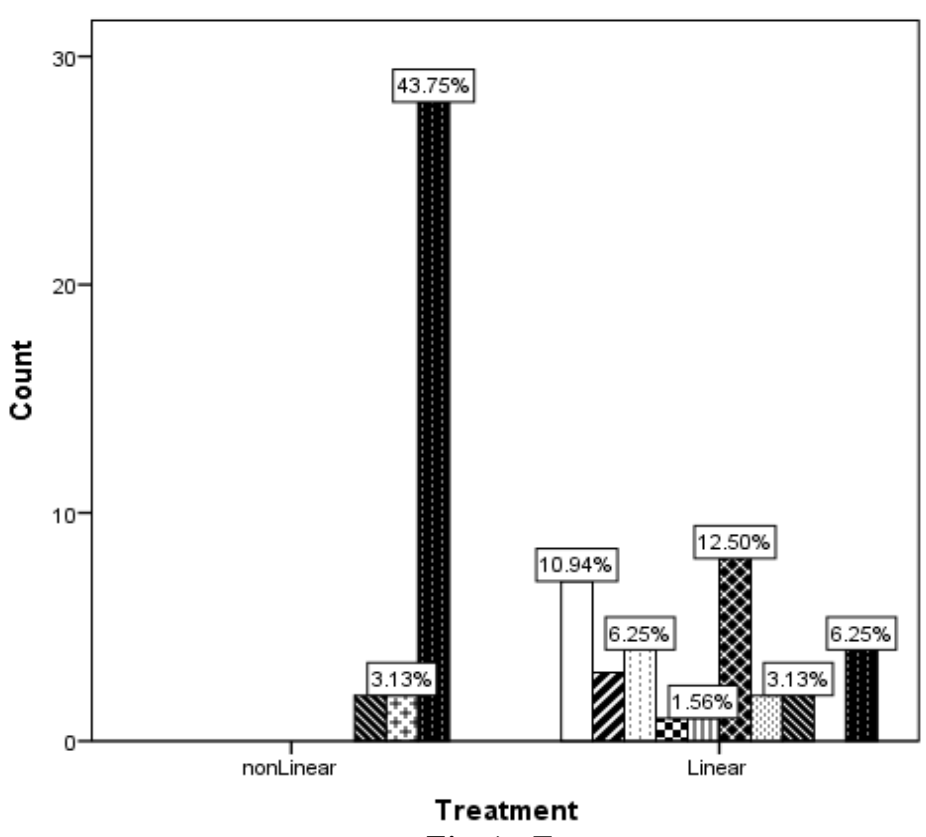

Contributions participants

Fig. 7: Treatment

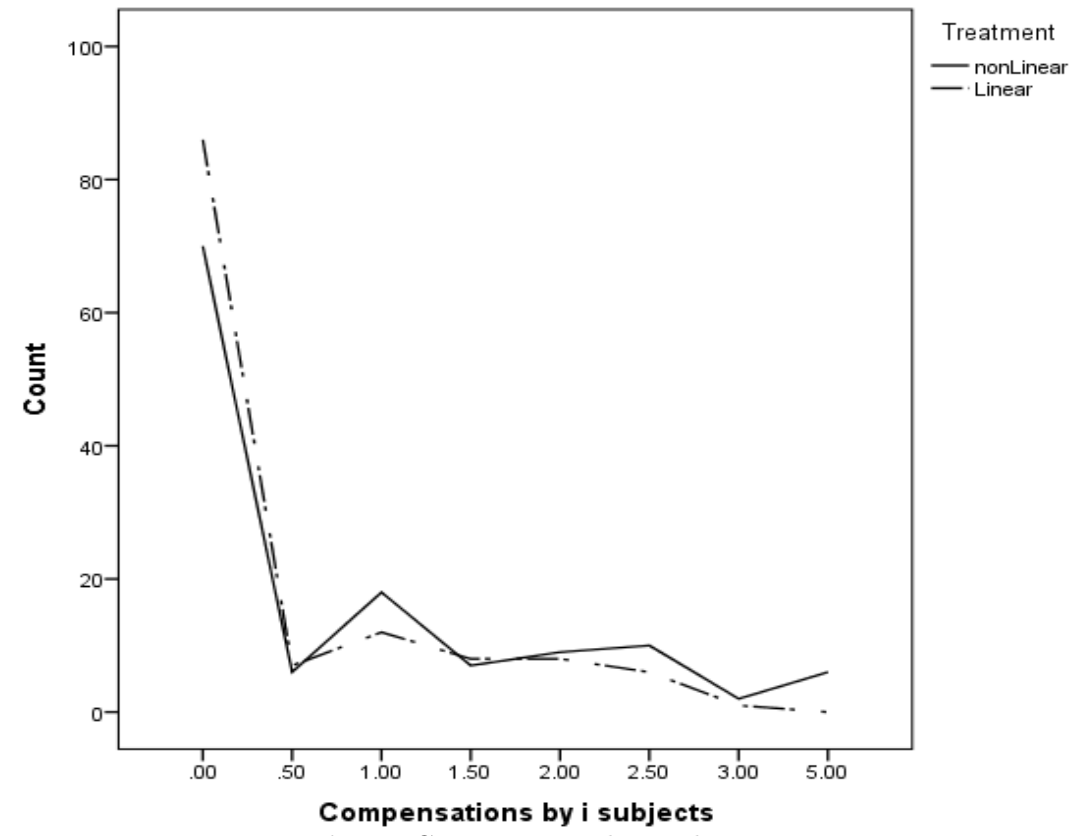

Fig. 8: Compensation by i subjects 


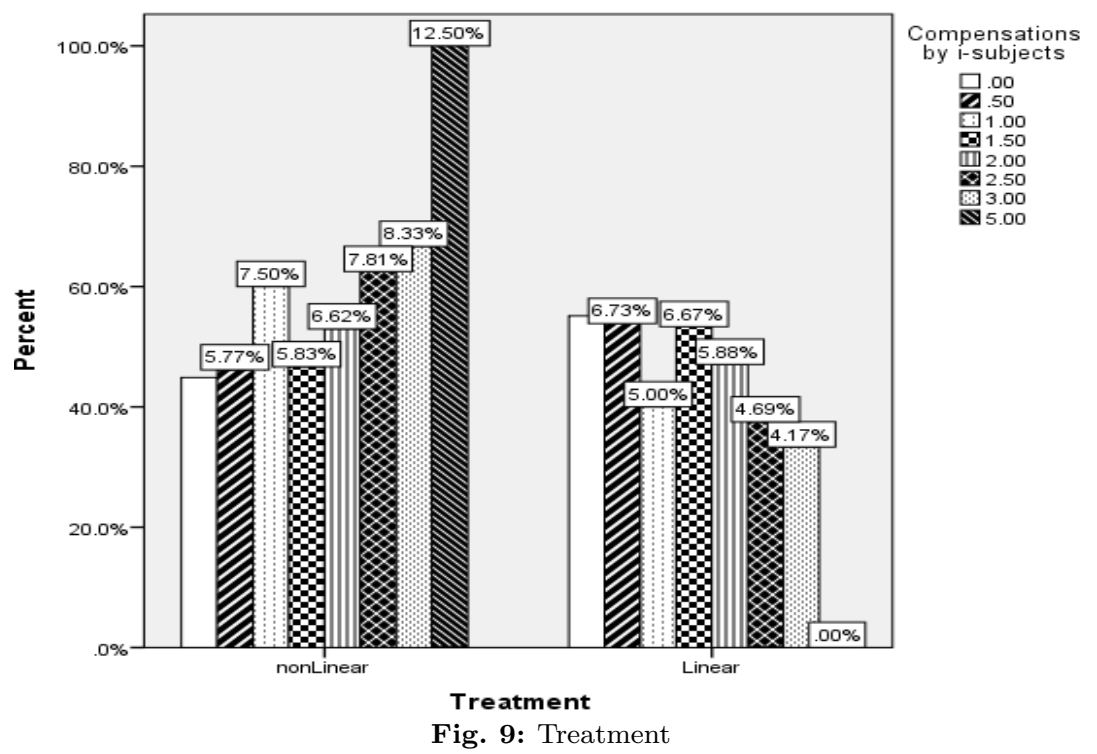

Table 1: Correlation between guilt behaviour, guilt repair, shame evaluation

\begin{tabular}{lcccc}
\hline & Guilt behavior & Guilt repair & $\begin{array}{l}\text { Shame } \\
\text { evaluation }\end{array}$ & $\begin{array}{l}\text { Shame } \\
\text { withdrawal }\end{array}$ \\
\hline Guilt behavior & 1 & & & \\
Guilt repair & 0.22 & 0.18 & & \\
Shame evaluation & $0.54^{* *}$ & 0.01 & 0.07 & -0.26 \\
Contribution by H in lin- & -0.06 & -0.18 & -0.08 & $-0.33^{*}$ \\
ear treatment & 0.00 & & & \\
Contribution by H in & & & & \\
non-linear treatment & & & & \\
\hline
\end{tabular}

